\title{
EBSD: Now Spanning Centimeters to Nanometers!
}

\author{
Joseph R. Michael ${ }^{1}$ and Jay Carroll ${ }^{2}$
}

1. Sandia National Laboratories Materials, Physical and Chemical Sciences, Albuquerque, NM.

2. Sandia National Laboratories, Materials Mechanics and Tribology, Albuquerque, NM.

Electron backscatter diffraction (EBSD) has now become an indispensable capability for understanding various phenomena in crystalline materials. Developments in EBSD hardware, software and techniques now allow crystallographic information to be efficiently collected over very large areas and enables data to be collected with step sizes as small as a few $\mathrm{nm}$ using transmission Kikuchi diffraction (TKD) [1,2]. Recent developments have produced systems that are capable of indexing over 3000 patterns each second. There have also been impressive developments in the use of EBSD for the qualitative and quantitative determination of strain [3]. With the addition of EBSD, the SEM now is one of the few tools that provides quantitative data of the morphology, chemistry and crystallography on length scales that span cm's to nm's. This paper will discuss the application of EBSD across length scales that spans 6 orders of magnitude. This range of length scales allows regions of interest to be located in large samples and then studied in detail at higher resolution.

A single crystal sample of Ta was rapidly deformed in compression along the $<110>$ axis in a Taylor cylinder impact test at a speed of $137.5 \mathrm{~m} / \mathrm{s}$. A cross section of the sample was metallographically prepared with a final vibratory polish using colloidal silica. An Oxford Symmetry EBSD system was used on a Zeiss Supra 55VP SEM operated at $20 \mathrm{kV}$ for mapping of the metallographic samples with a step size of $5 \mu \mathrm{m}$ and $0.4 \mu \mathrm{m}$. TKD was performed with the Oxford Symmetry EBSD system or a Bruker Optimus (on-axis EBSD camera) on a Zeiss Gemini 500 SEM operated at $30 \mathrm{kV}$ with a $4 \mathrm{~nm}$ step size. The thin sample was prepared by in-situ lift-out with a Thermo Scientific Helios 660/G3 DualBeam using standard sample preparation techniques.

Figure 1a is a montaged EBSD inverse pole figure (IPF) map of the $27 \mathrm{~mm}$ long test sample. This large low-resolution map was critical to understand the overall deformation that occurred and it is needed to locate areas for further study. Figure $2 b$ is a much higher resolution map of an area of interest where extensive deformation has occurred during the compression experiment. Collection of higher resolution EBSD maps allows many of the details in this region to be better visualized. The features observed in Figure $1 \mathrm{~b}$ are shown in the channeling contrast image shown in Figure 1c at a much smaller field of view. An in-situ FIB thin section was produced normal to the linear features as shown by the line in Figure 1c. The resulting TKD misorientation map acquired with a step size of $4 \mathrm{~nm}$ is shown in Figure $1 \mathrm{~d}$. This map is displayed as the misorientation with respect to an undeformed region of the sample.

This example shows the extreme length scale range over which EBSD can be applied. Maps that cover large areas are extremely helpful when possibly heterogeneous microstructures or deformation are possible. Latest generations of EBSD cameras and software allow large maps as shown in Figure 1a to be acquired in less than 5 hours thus improving the utilization of the SEM and providing information that cannot be obtained from other methods. Once the over view type map is obtained detailed orientation information can be collected from targeted regions of the sample [4]. 


\section{References:}

[1] J Goulden et al, Microscopy and Microanalysis 20 (2014), p. 982.

[2] RR Keller and RH Geiss, Journal of Microscopy 245 (2012), p. 245.

[3] AJ Wilkinson and TB Britton, Materials Today 15 (2012), p. 366.

[4] Sandia National Laboratories is a multimission laboratory managed and operated by National Technology and Engineering Solutions of Sandia, LLC, a wholly owned subsidiary of Honeywell International, Inc., for the U.S. Department of Energy's National Nuclear Security Administration under contract DE-NA0003525.
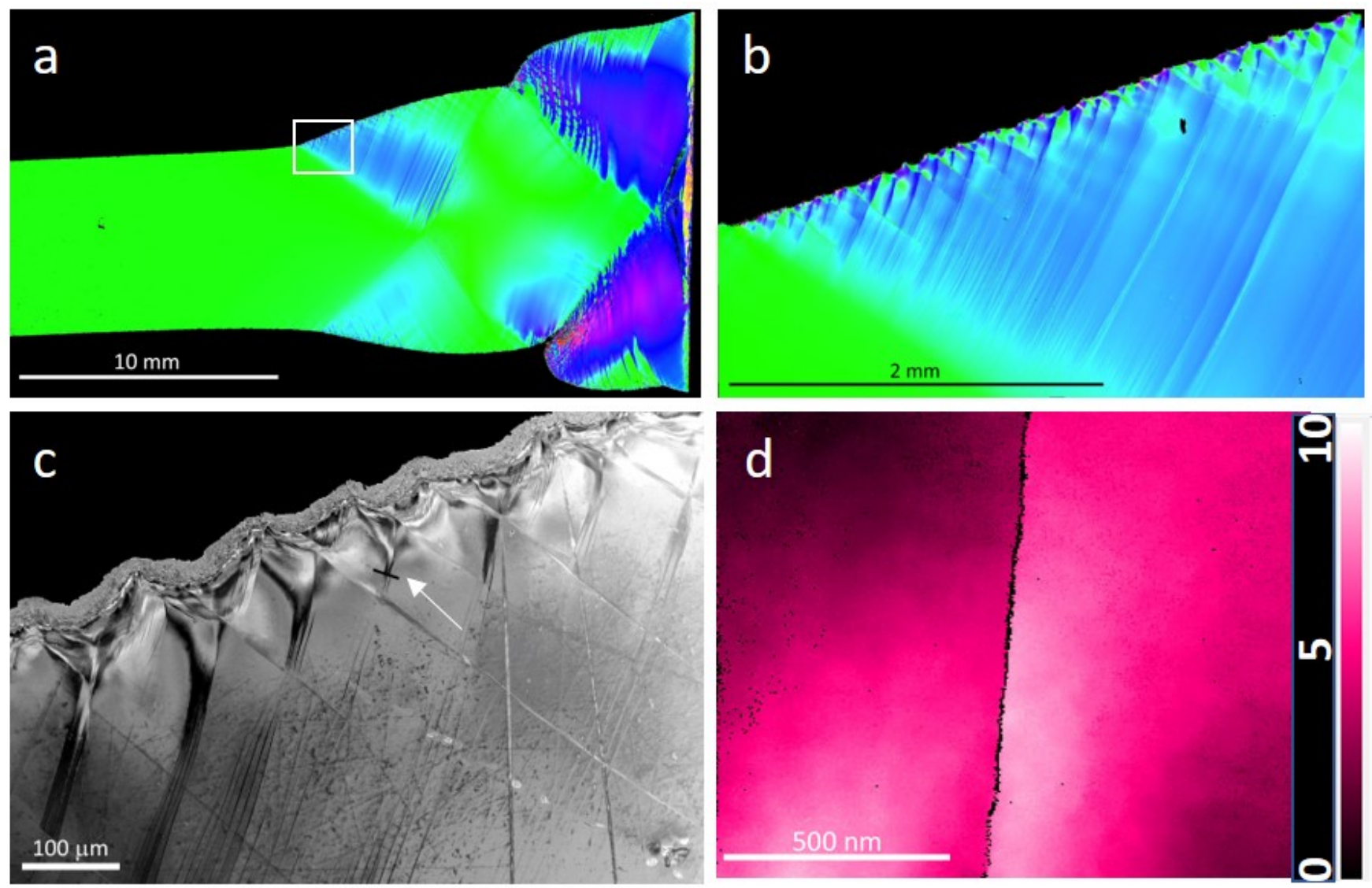

Figure 1. EBSD, channeling contrast and TKD images acquired from the deformed Ta sample. a) Large area montaged inverse pole figure EBSD map with respect to the long axis of the cylinder acquired with a $5 \mu \mathrm{m}$ step size at 952 patterns/ second. b) Montaged EBSD IPF map of the boxed region in Figure 1a acquired with a $0.4 \mu \mathrm{m}$ step size at 2200 patterns per second (about 58M pixels), c) Channeling contrast image of a region in Figure 1b, small bar next to the arrow represents the FIB sample location d) TKD of the FIB lift out sample shown as a misorientation map with respect to the undeformed Ta single crystal of a single slip feature acquired with a $4 \mathrm{~nm}$ step size. White to black color scale represents $10^{\circ}$ orientation change with respect to the undeformed region. 